\title{
Correction to: Short Text Clustering with a Deep Multi-embedded Self-supervised Model
}

Kai Zhang, Zheng Lian, Jiangmeng Li, Haichang Li, and Xiaohui Hu

Correction to:

Chapter "Short Text Clustering with a Deep Multi-embedded Self-supervised Model" in: I. Farkaš et al. (Eds.): Artificial Neural Networks and Machine Learning - ICANN 2021, LNCS 12895, https://doi.org/10.1007/978-3-030-86383-8_12

Due to an oversight, the second affiliation of three co-authors was omitted in the originally published version. The revised version has the correct affiliations of all co-authors. 